\title{
Photon Management
}

Frank Wyrowski

Chair/Editor

\section{7-28 April 2004}

Strasbourg, France

Sponsored and Published by

SPIE-The International Sociefy for Optical Engineering

Cooperating Organizations

EOS-European Optical Society (United Kingdom)

SFO-Société Française d'Optique (France)

SIOF-Società Italiana di Ottica e Fotonica (Italy)

DGaO-Deutsche Gesellschaft für angewandte Optik (Germany)

IEE-The Institution of Electrical Engineers

Photonics Clusters (United Kingdom)

oemagazine

NEMO-Network of Excellence in Micro-Optics

Supported by

Région Alsace (France)

Communauté Urbaine de Strasbourg (France)

Conseil Général du Bas-Rhin (France)

Alsace Development Agency (France)

European Office of Aerospace Research and Development,

U.S. Air Force Office of Scientific Research 


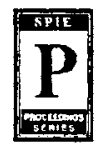

The papers included in this volume were part of the technical conference cited on the cover and title page. Papers were selected and subject to review by the editors and conference program committee. Some conference presentations may not be available for publication. The papers published in these proceedings reflect the work and thoughts of the authors and are published herein as submitted. The publisher is not responsible for the validity of the information or for any outcomes resulting from reliance thereon.

Please use the following format to cite material from this book:

Author(s), "Title of Paper," in Photon Management, edited by Frank Wyrowski, Proceedings of SPIE Vol. 5456 (SPIE, Bellingham, WA, 2004) page numbers.

ISSN 0277-786X

ISBN 0-8194-5383-8

Published by

SPIE-The International Society for Optical Engineering

P.O. Box 10, Bellingham, Washington 98227-0010 USA

Telephone $1360 / 676-3290$ (Pacific Time)- Fax $1360 / 647-1445$

http://www.spie.org

Copyright (c) 2004, The Society of Photo-Optical Instrumentation Engineers

Copying of material in this book for internal or personal use, or for the internal or personat use of specific clients, beyond the fair use provisions granted by the U.S. Copyright Law is authorized by SPIE subject to payment of copying fees. The Transactional Reporting Service base fee for this volume is $\$ 15.00$ per article (or portion thereof), which should be paid directly to the Copyright Clearance Center (CCC), 222 Rosewood Drive, Danvers, MA 01923. Payment may also be made electronically through CCC Online at http://mww.copyright.com. Other copying for republication, resale, advertising or promotion, or any form of systematic or multiple reproduction of any material in this book is prohibited except with permission in witing from the publisher. The CCC fee code is 0277 $786 \times 104 / \$ 15.00$.

Printed in the United States of America. 


\title{
Comparison of electromagnetic theories to predict the efficiencies of the different orders inside a volume grating
}

\author{
C. Neipp ${ }^{* a}$, C. Pascual ${ }^{\mathrm{a}}$, A. Márquez ${ }^{\mathrm{a}}$, M. L. Álvarez ${ }^{\mathrm{a}}$, I. Pascual ${ }^{\mathrm{b}}$ and A. Beléndez ${ }^{\mathrm{a}}$ \\ ${ }^{a}$ Departamento de Física, Ingeniería de Sistemas y Teoría de la Señal, Universidad de Alicante Ap. \\ 99, Alicante, Spain E-03080; \\ ${ }^{b}$ Departamento Interuniversitario de Óptica, Universidad de Alicante, Ap. 99, Alicante, Spain E- \\ 03080
}

\begin{abstract}
In this work we make a comparative study between different theories to predict the efficiencies of the different orders that propagate inside a volume phase grating. For the case of a pure sinusoidal grating, transmission and reflection, the theories of Rigorous Coupled Wave Theory (RCW), Coupled Wave Theory (CW) and Kogelnik's Theory are compared. This comparison allows establishing the range of physical values where the more approximate theories, $\mathrm{CW}$ and Kogelnik's Theory are applicable. On the other hand for the case of a general dielectric grating, transmission and reflection, the RCW, CW, and a thin matrix decomposition method (TMDM) are also compared. The theoretical study is also validated by comparing the theoretical results with experimental data obtained in volume phase diffraction gratings recorded on photographic emulsions. To record the volume phase diffraction gratings BB-640 emulsions were exposed to an interference pattern of light from a He-Ne laser $(633 \mathrm{~nm})$.
\end{abstract}

Keywords: Holography; Volume gratings, Holographic recording materials, Photographic emulsions

\section{INTRODUCTION}

Volume diffraction gratings have been an interesting field of application during the last decades. Although there is a great understanding of how light propagates inside different periodic structures, the field of study of electromagnetic theories to accurately predict the behaviour of waves inside volume holograms is still interesting. An usual way to calculate the efficiencies of the different orders that propagate in the volume grating is to solve Maxwell equations for the case of an incident plane wave on a medium which the relative dielectric permittivity varies in ${ }^{1,2}$. Although the idea seems clear and precise, there are in the literature a great number of models that allow solving the problem.

The most popular theory in holography that has provided an analytical solution for the efficiency of the first and zero order is the Coupled Wave Theory of Kogelnik ${ }^{3}$. During decades researchers in the field of holography have used the analytical expressions deduced by Kogelnik to estimate the theoretical predictions of phase and amplitude, transmission and reflection volume holograms. Kogelnik assumed that only two orders propagated in the hologram, orders zero and +1 , and obtained analytical solutions for the efficiencies of the first and zero order when a plane wave impinges on a diffraction grating with a sinusoidal variation of its electro-optical properties (relative dielectric permittivity and conductivity). The highly predictive character of the expressions derived by Kogelnik made his work one of the most cited by holographic researchers. Nonetheless Kogelnik's theory assumed some approximations that make it inaccurate for some cases, such as dielectric gratings that are not sinusoidal or for thin gratings (outside the Bragg regime). In these cases more rigurous theories are needed, such as the rigurous coupled wave theory proposed by Moharam and Gaylord ${ }^{4}$. Since the first introduction of the Rigorous Coupled Wave (RCW) theory to predict the efficiency of the different orders that propagate inside a hologram, a lot of advances have been done in the research of electromagnetic theory applied to periodic structures ${ }^{5-9}$. The Rigorous Coupled Wave Theory has been applied with success to volume holograms ${ }^{10}$, photonic band structures ${ }^{11}$, diffractive lenses ${ }^{12}$, etc. And it is also the method that should be used to test the validity of

*ristian@dfists.ua.es; phone: +34-96-5903651: Fax: +34-96-5909750 
the different approximations that have been done and are still doing in order to obtain analytical functions for the efficiencies of the different orders that propagate in the hologram. In particular, in this work we will compare the CW, $\mathrm{RCW}$ and Kogelnik's theories to study the range validity of Kogelniks' expressions.

Although exact predictions can be obtained by using the RCW it is still interesting to find analytical expressions in order to calculate the efficiency of the different orders that propagate inside the hologram in different situations. Analytical expressions give a deeper understanding of the physical processes than numerical solutions do. In addition, by direct inspection of the analytical expressions a clearer interpretation of how the different parameters influence the efficiency of the different orders is got. As commented, Kogelnik"s theory can be applied to predict the efficiency of the first order for sinusoidal diffraction gratings near first Bragg replay angular condition. To obtain analytical expressions for the efficiencies of the first and second order at second on-Bragg replay angular condition other methods are needed. Using a thin matrix decomposition method (TMDM), Alferness ${ }^{13}$ obtained analytical expressions for the zero, first and second orders when the volume hologram is reconstructed at second on-Bragg replay angular condition. In this work we will also present a comparison between the TMDM and the RCW theory to study the range of validity of this theory.

\section{RIGUROUS COUPLED WAVE AND COUPLED WAVE THEORY}

In this section we will explain the basic assumptions of the coupled wave theories to study the propagation of light inside a phase sinusoidal transmission grating, where the relative permittivity in the hologram can be expressed as:

$$
\varepsilon_{r}(x, z)=\varepsilon_{r 0}+\varepsilon_{r 1} \cos \boldsymbol{K} \cdot \boldsymbol{r}
$$

$\varepsilon_{\mathrm{r} 0}$ is the average dielectric constant, $\varepsilon_{\mathrm{T} 1}$ the amplitude of the relative permittivity and $\boldsymbol{K}$ is the grating vector, which is related to the period of the interference fringes, $A$, as follows:

$$
|\boldsymbol{K}|=2 \pi / \Lambda
$$

The electric field inside the hologram is supposed to be an infinite sum of orders in the form ${ }^{1,2}$ :

$$
E_{1}=\sum_{i} S_{i}(z) \exp \left(-j \rho_{i} \cdot \boldsymbol{r}\right) \quad \mathrm{i}=0, \pm 1, \pm 2, \ldots
$$

$S_{\mathrm{i}}$ and $\rho_{1}$ are the amplitude and the propagation vector of the $\mathrm{i}^{\text {th }}$ diffracted order, respectively. The propagation vector is related to the grating vector as:

$\rho_{0}$ being the propagation vector of the incident wave.

$$
\rho_{\mathrm{i}}=\rho_{0}+i K
$$

Substitution of equations (1), (3) and (4) in the Helmholtz equation yields the following set of equations:

$$
\frac{j}{2 \beta} \frac{d^{2} S_{i}(z)}{d z^{2}}+C_{i} \frac{d S_{i}(z)}{d z}-j \kappa \Omega i(i+P) S_{i}(z)+j \kappa\left(S_{i+1}(z)+S_{i-1}(z)\right)=0
$$

$C_{\mathrm{i}}$ are the called obliquity factors and are the cosine of the angles that the propagation vectors of the different orders form with the $\mathrm{z}$ axis. In the particular case of non-slanted diffraction gratings, $C_{\mathrm{i}}=\cos \theta_{0}$, where $\theta_{0}$ is the angle of reconstruction inside the medium

$\beta$ is the propagation constant inside the hologram,

$$
\beta=\frac{2 \pi}{\lambda}\left(\varepsilon_{r 0}\right)^{1 / 2}
$$

$\lambda$ is the wavelength of light in vacuum.

The parameter $\kappa$ is the coupling constant, defined as:

$$
\kappa=\frac{\beta \varepsilon_{r 1}}{4 \varepsilon_{r 0}}
$$

The $\Omega$ parameter is: 


$$
\Omega=\frac{|\boldsymbol{K}|^{2}}{2 \beta \kappa}
$$

Finally, the parameter $P$ is defined as:

$$
P=\frac{2 \beta}{|\boldsymbol{K}|} \sin \theta_{0}
$$

The parameter $P$ is the called impact parameter and takes the values, $P=1$ for reconstruction at first Bragg angle, $P=2$ at the second and so on.

Till the publication of Moharam and Gaylord 's work in $1982^{4}$ the coupled wave equations (5) were solved by neglecting the second derivatives of it. This method assumes that there is slow transference of energy from one order to the others, and we will refer to it as CW (coupled wave theory) approximation. The work published by Moharam and Gaylord allowed to solve rigorously equations (5), and we will call this method RCW. In this work we will demonstrate that in the range of parameters analyzed, neglecting the second derivatives of equation (5) does not almost affect the final results.

Once equations (5) are solved the diffraction efficiencies of the different orders are calculates as:

$$
D E_{\mathrm{i}}=S i(d) S i(d)^{*}
$$

\section{KOGELNIK'S COUPLED WAVE THEORY}

If only two orders are supposed propagating inside the holographic medium and equation (5) is used, Kogelnik's expressions for the efficiency of the zero and first order can be derived ${ }^{3}$. The amplitudes of the zero, $R(z)$, and first order, $S(z)$, obtained by Kogelnik at the output of the hologram $(z=d)$ are:

$$
\begin{gathered}
R(d)=\exp \left(-j \xi-\frac{\alpha d}{2 c_{r}}\right)\left[\cos \Phi+j\left(\frac{\xi}{\Phi}\right) \sin \Phi\right] \\
S(d)=-j\left(\frac{c_{r}}{c_{s}}\right)^{1 / 2} \exp \left(-j \xi-\frac{\alpha d}{2 c_{r}}\right) \frac{\sin \Phi}{\Phi / \nu}
\end{gathered}
$$

where $c_{\mathrm{r}}=\cos \theta_{\mathrm{r}}$ and $\mathrm{c}_{\mathrm{s}}=\cos \theta_{\mathrm{d}}, \theta_{\mathrm{r}}$ and $\theta_{\mathrm{d}}$ are the angles that the transmitted and diffracted propagation vectors form with the normal of the hologram. $\alpha$ is the absorption constant and $d$ is the thickness of the hologram. The following parameters are also defined:

and

$$
\begin{gathered}
\Phi=\sqrt{v^{2}+\xi^{2}} \\
v=\frac{\pi \Delta n d}{\lambda \cos \theta^{\prime}}
\end{gathered}
$$

$$
\begin{gathered}
\xi=j \frac{d}{2}\left(\frac{\alpha}{c_{r}}-\frac{\alpha}{c_{s}}-j \frac{\vartheta}{c_{s}}\right) \\
\vartheta=\frac{\beta^{2}-\sigma^{2}}{2 \beta}
\end{gathered}
$$

$\Delta n$ is the refractive index modulation. $\theta^{\prime}$ is the angle of reconstruction in the recording medium related to the angle at reconstruction in air by Snell's law. whereas $\beta$ and $\sigma$ are the propagation constants of the transmitted and diffracted waves, respectively.

The diffraction efficiency can be expressed as:

$$
\eta=\frac{\left|c_{s}\right|}{c_{r}} S S^{*}
$$


and the transmission efficiency as:

$$
\tau=R R^{*}
$$

The final expressions of $\eta$ and $\tau$ as a function of $v$ and $\xi$ are:

$$
\begin{gathered}
\eta=\exp \left(-\alpha d / \cos \theta^{\prime}\right) \frac{\sin ^{2}\left(v^{2}+\xi^{2}\right)^{1 / 2}}{1+\left(\xi^{2} / v^{2}\right)} \\
\tau=\exp \left(-\alpha d / \cos \theta^{\prime}\right)\left[1-\frac{\sin ^{2}\left(v^{2}+\xi^{2}\right)^{1 / 2}}{1+\left(\xi^{2} / v^{2}\right)}\right]
\end{gathered}
$$

\section{ALFERNESS EXPRESSION OF THE EFFICIENCY OF THE SECOND ORDER ON SECOND ON-BRAGG REPLAY ANGULAR CONDITION}

As commented, Kogelnik's theory is only applicable to sinusoidal diffraction gratings. If a diffraction grating with higher harmonics in the refractive index is studied, other methods are needed to obtain the efficiencies of the different orders that propagate inside the grating.In this section the expression obtained by Alferness using a TMDM for the efficiency of the first and second orders at second on-Bragg angular replay condition will be introduced. For a complete derivation of the formula see ref. 13. The refractive index stored in an unslanted phase transmission hologram can be expressed through equation (21) if only three harmonic components of the refractive index are considered:

$$
n(\mathrm{x})=n_{0}+n_{1} \cos (2 \pi f x)+n_{2} \cos (4 \pi f x)
$$

where $f$ is the spatial frequency of the transmission diffraction grating, related to the modulus of the grating vector, $K$, as:

$$
K=2 \pi f
$$

$n_{0}$ is the average refractive index of the holographic medium and $n_{1}$ and $n_{2}$ are the first and second harmonic components of the refractive index, respectively.

The expressions obtained by Alferness for the efficiencies of the first, $\eta_{1}$, and second, $\eta_{2}$, orders when reconstruction of the hologram is done at second on-Bragg replay angular condition are:

$$
\eta_{1}=\left(\gamma_{1}^{2} / \chi^{2}\right) \sin ^{2} \chi
$$

and

$$
\left.\eta_{2}=1 / 4\left[\cos \chi-\cos \left(3 \gamma_{2} / 2+\xi\right)\right]^{2}+\left[(\rho / \chi) \sin \chi-\sin \left(3 \gamma_{2} / 2+\xi\right)\right]^{2}\right\}
$$

$\gamma_{1}$ and $\gamma_{2}$ are the fundamental and harmonic grating strengths which can be expressed as:

The following parameters are also defined:

$$
\begin{gathered}
\gamma_{1}=\pi n_{1} d / \lambda\left(c_{1} c_{0}\right)^{1 / 2} \\
\gamma_{2}=\pi n_{2} d / \lambda c_{0}
\end{gathered}
$$

$$
\begin{gathered}
\xi=\left(\pi \lambda / n_{0}\right) f^{2} d\left(1+\cos \theta_{2 B}\right)^{-1} \\
\rho=\xi-\gamma_{2} / 2 \\
\chi=\left(\rho^{2}+2 \gamma_{1}^{2}\right)^{1 / 2}
\end{gathered}
$$

In equations (23)-(29), $\lambda$ is the wavelength in air, $c_{0}$ and $c_{1}$ the cosines of the angles formed by the zero and first order propagation vectors with the normal of the hologram, respectively, and $\theta_{2 \mathrm{~B}}$ is the angle of reconstruction in air at second on-Bragg replay angular condition. which is related to the spatial frequency, $f$, through:

$$
f=\left(n_{0} / \lambda\right) \sin \theta_{2 B}
$$

In Sec. 5 the validity of expressions (23).(24) will be checked by using the RCW theory. 


\section{COMPARISON OF RCW, CW AND KOGELNIK'S THEORY}

At first we will compare the theoretical results obtained by using Kogelnik's Coupled Wave Theory for the efficiency of the first order at first on-Bragg replay angular condition with the results obtained using the RCW and CW theories ${ }^{14}$. In order to make the comparison as realistic as possible we considered in the theoretical simulations a relatively easy achievable value of the refractive index in some usual materials, $n_{l}=0.025$. This value can be recorded in photographic emulsions, dichromated gelatins or silver halide sensitized gelatins ${ }^{15}$, for instance. The range of thickness values used in the simulations was of $[0 \mu \mathrm{m}, 18 \mu \mathrm{m}]$. In addition 9 orders were also retained in the calculations for equations $(5): \mathrm{N}= \pm$ $\mathrm{i}, \mathrm{i}=0,1,2, \ldots$ Two spatial frequencies were also chosen: 350 lines $/ \mathrm{mm}$ and 750 lines $/ \mathrm{mm}$.

It should be noticed that $\mathrm{CW}$ and $\mathrm{RCW}$ theories assume a harmonic dependence of the relative permitivity, whereas experimental researchers in Holography usually describe the phase grating in terms of the refractive index. Therefore, it is necessary to relate the average and first harmonic component of the refractive index to the average and first harmonic component of the dielectric permittivity. Using:

$$
\varepsilon_{1}=2 n_{0} n_{1}
$$

where $n_{0}$ is the average refractive index and $n_{I}$ is the refractive index modulation. Equation (31) holds whenever $n_{l}<<n_{0}$. In the theoretical calculations a value of $n_{0}=1.63$ was used, which is a practical value for materials such as photographic emulsions.

Figs. 1 and 2 show the efficiency of the first order, +1 , as a function of the thickness, $d$, under Bragg condition for a transmission diffraction grating with two spatial frequencies of 350 and 750 lines $/ \mathrm{mm}$. It can be seen that for a spatial frequency of 350 lines/mm the theoretical simulations obtained by using the $\mathrm{CW}$ and $\mathrm{RCW}$ method differ clearly from that obtained by using Kogelnik's Theory. The value of $\Omega$ from equation (8) was of 1.20 for the grating considered in Fig. 1. For such a low spatial frequency it is clear that multi-wave diffraction occurs and Kogelnik's Theory is not applicable. Nonetheless, it is interesting to notice that good agreement between $\mathrm{CW}$ and RCW simulations is obtained, justifying the neglect of the second derivatives in this case.

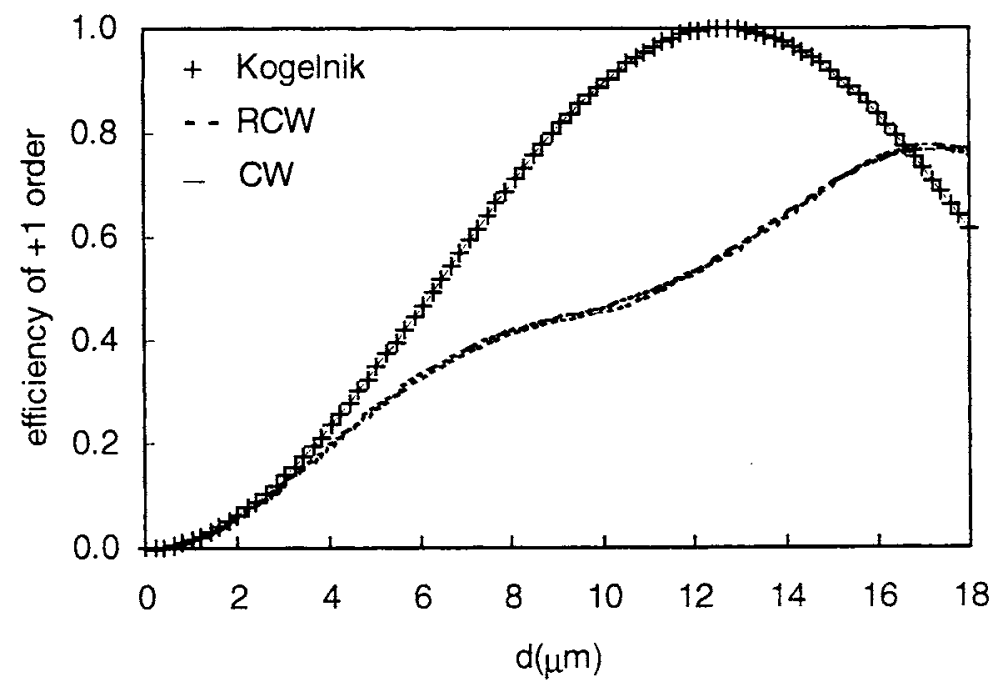

Fig.1. Efficiency of the first order as a function of the thickness, $d$. under Bragg condition for a transmission diffraction grating of 350 lines $/ \mathrm{mm}$ with a refractive index modulation of $n_{l}=0.025$.

The results obtained, using a spatial frequency of 750 lines/mm (Fig. 2). are clearly different. In this case Kogelnik's, $\mathrm{CW}$ and RCW Theories all agree, being the value of $\Omega, 5.53$ in this case. 
Finally we want to present a comparison between theoretical and experimental results for a diffraction grating recorded on a photographic emulsion with a spatial frequency of $1200 \mathrm{lines} / \mathrm{mm}$ (Fig. 3). In this case the refractive index recorded in the photographic emulsion was of, 0.099 , which is a relatively large value. Nonetheless, good agreement between the results obtained using Kogelnik and RCW can be observed.

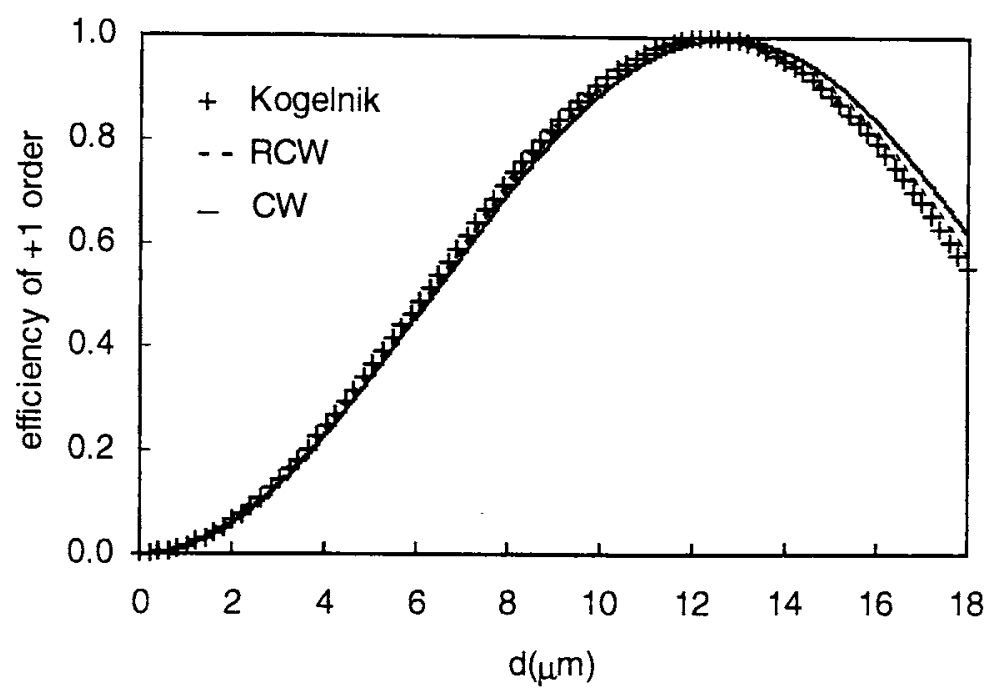

Fig.2. Efficiency of the first order as a function of the thickness, $d$, under Bragg condition for a transmission diffraction grating of 750 lines/mm with a refractive index modulation of $n_{l}=0.025$.

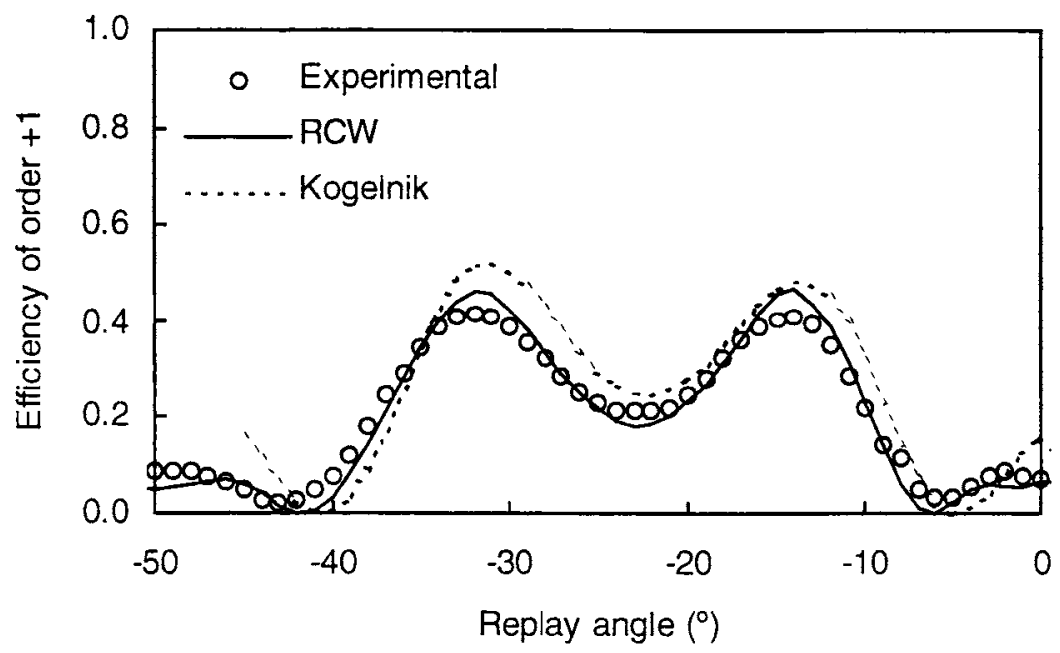

Fig. 3. Efficiency of the first order as a function of the reconstruction angle for a transmission diffraction grating of $1200 \mathrm{lines} / \mathrm{mm}$ recorded on BB-640 emulsion and presenting a refractive index modulation of $\mathrm{n} 1=0.099$ 


\section{COMPARISON OF RCW, CW AND TMDM FOR THE EFFICIENCY OF THE FIRST AND SECOND ORDER AT SECOND ON-BRAGG REPLAY ANGULAR CONDITION}

As commented, Kogelnik's theory has been largely used in Holography to predict the efficiency of the first and zero order for sinusoidal volume diffraction gratings. Nevertheless, it cannot be applied to diffraction gratings that present higher harmonics in the Fourier expansion of the refractive index. The importance of considering these higher harmonics has been demonstrated in photopolymer materials, for instance. Different models have been proposed in the literature to explain the mechanism of hologram formation in photopolymer materials ${ }^{16-23}$, and the great majority of them predict the existence of higher harmonics in the Fourier expansion of the polymer concentration and, therefore, higher harmonics in the refractive index. Since Kogelnik's theory is not applicable in this cas $\mathrm{e}^{24}$, new methods should be used to obtain analytical expressions for the efficiency of the different orders propagating inside the grating. The TMDM method proposed by Alferness, served to obtain the efficiencies of the zero, first, and second order when a plane wave impinges onto a diffraction grating at second on-Bragg angular replay condition. In this section we will compare the results obtain by using the RCW and expressions (23) and (24) ${ }^{25}$.

In the same manner we did in Sec. 5 an appropriated conversion of the parameters is made, since the properties of the material is described in terms if $n$ for the TMDM method, and in terms of $\varepsilon$ in the RCW theory. Since we will take into account only the zero, first and second harmonic components of the refractive index the refractive index and relative dielectric permittivity can be expressed as:

$$
\begin{gathered}
n(x, z)=\sum_{h=0}^{2} n_{h} \cos (i K x) \\
\varepsilon_{r}(x, z)=\sum_{h=-2}^{2} \varepsilon_{h} \exp (j h K x)
\end{gathered}
$$

It is easy to relate the harmonic components of the refractive index to the Fourier components of the dielectric permittivity of equation, provided that

It can be found that

$$
n_{1}, n_{2}<<n_{0}
$$

and

$$
\varepsilon_{ \pm i}=n_{0} n_{i} \quad \text { for } \mathrm{i}=1,2
$$

$$
\varepsilon_{r 0}=n_{0}^{2}
$$

Fig. 4 represents the efficiency of the first and second order as a function of the fundamental grating strength for a value of the second harmonic component of the refractive index, $n_{2}=n_{1} / 5$, for a volume diffraction grating with a spatial frequency of 350 lines $/ \mathrm{mm}$. The value of the first harmonic component, $n_{1}$, of the diffraction grating used was of 0.035 , which is a physically achievable value for different recording materials such as photographic emulsions, dichromated gelatins, silver halide sensitized gelatins, etc. The dashed line corresponds to the analytical expression (3), whereas the continuous line corresponds to the simulation done using the RCW. The disagreement between both curves for the second order, is due to the fact that for such a low spatial frequency multi-wave diffraction occurs ${ }^{1,2}$, that is, more than three orders propagate inside the hologram. Therefore, in this case, the approximate solution of Alferness doesn't seem to work well for this low frequency.

Fig. 5 shows the efficiency of the first and second order as a function of the grating strength for a spatial frequency of 1000 lines $/ \mathrm{mm}$ for the same value of the second order of the refractive index. In this case there is better agreement between both theories, but the curves corresponding to the analytical expressions (23)-(24) take higher values than those obtained using the rigorous coupled wave theory. This is due to the fact that in the case of the RCW method 9 orders were included whereas in the case of the TMDM only 3 were assumed. Although there is low coupling between the second order and higher ones, neglecting this power transfer, such as done by the method proposed by Alferness. will conduct to higher efficiencies of the second order. 


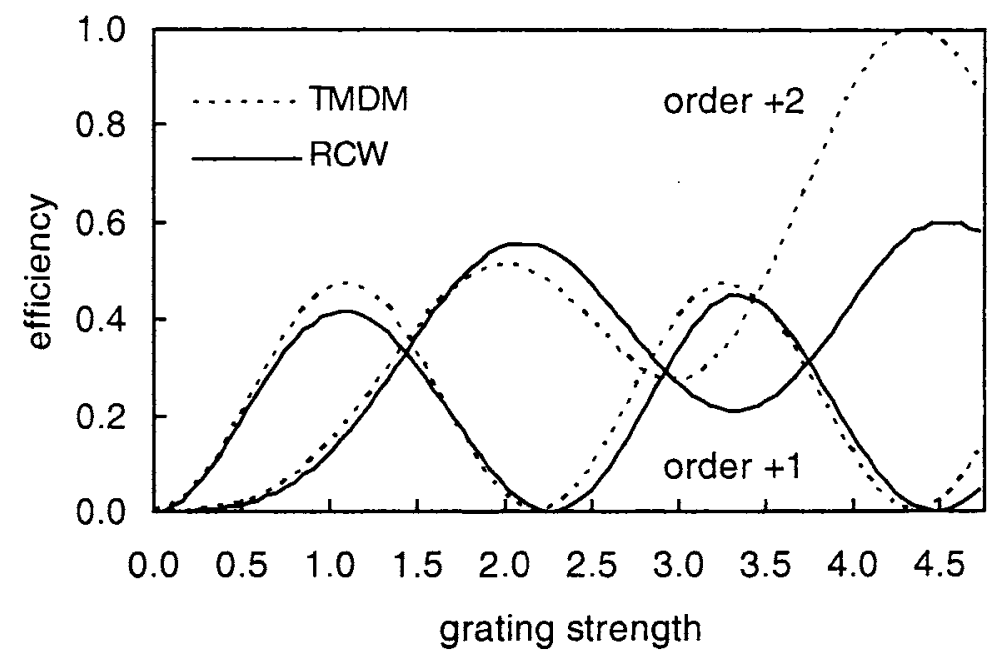

Fig. 4. Efficiency of the first and second order as a function of the fundamental grating strength for a transmission diffraction grating of 350 lines $/ \mathrm{mm}$. The value of the second harmonic component of the refractive index was of $n_{2}=n_{1} / 5$, and $n_{1}=0.035$.

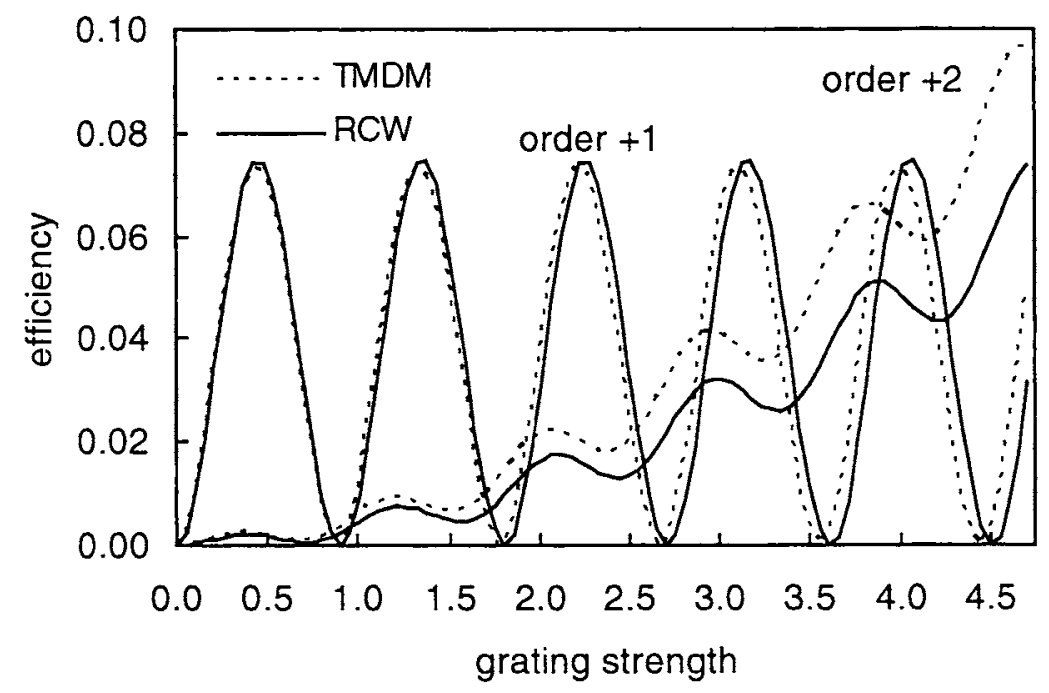

Fig. 5. Efficiency of the first and second order as a function of the fundamental grating strength for a transmission diffraction grating of 1000 lines $/ \mathrm{mm}$. The value of the second harmonic component of the refractive index was of $n_{2}=n_{1} / 5$, and $n_{1}=0.035$.

\section{CONCLUSIONS}

In this work a comparative study between different theories to predict the efficiencies of the different orders that propagate inside a volume phase grating is made. For the case of a pure sinusoidal grating, transmission and reflection, the theories of Rigorous Coupled Wave Theory, Coupled Wave Theory and Kogelnik's Theory have been compared. It has been demonstrated that for transmission diffraction gratings recorded in materials of the photographic emulsion type (refractive index modulations $\sim 0.025$ ) and for spatial frequencies higher than 750 lines $/ \mathrm{mm}$ the analytical expressions of Kogelnik yield to similar values as those obtained the $\mathrm{CW}$ and $\mathrm{RCW}$ methods. 
On the other hand the analytical expressions for the efficiency of the first and second order for an unslanted transmission diffraction grating, obtained by using a TMDM method proposed by Alferness have been compared with the results obatined using the RCW. It has been shown that for spatial frequencies higher than 750 lines $/ \mathrm{mm}$ the expressions provided by Alferness agree quite well with the results obtained by using the Rigurous Coupled Wave Theory. This justifies the validity of Alferness expression in the range of parameteres studied.

\section{ACKNOWLEDEGMENTS}

This work was supported by the Ministerio de Ciencia y Tecnologia, CICYT, Spain, under project MAT2000-1361C04-04. 04.

\section{REFERENCES}

1. L. Solymar and D. J. Cooke, Volume Holography and Volume Gratings, Academic, London, 1981.

2. R. R. A. Syms, Practical Volume Holography, Clarendon Press, Oxford, 1990.

3. H. Kogelnik, "Coupled wave theory for thick hologram gratings", Bell Systems Technology Journal, 48, 2909-2947, 1969.

4. M. G. Moharam and T. K. Gaylord, "Rigurous coupled-wave analysis of planar-grating diffraction", Journal of the Optical Society of America, 71, 811-818, 1981.

5. M. G. Moharam and T. K. Gaylord, "Rigurous coupled-wave analysis of planar-grating diffraction- $E$-mode polarization and losses", Journal of the Optical Society of America, 73, 451-455, 1983.

6. M. G. Moharam and T. K. Gaylord, "Three dimensional vector coupled-wave analysis of planar-grating diffraction", Journal of the Optical Society of America, 73, 1105-1112, 1983.

7. T. K. Gaylord and M. G. Moharam, "Analysis and applications of optical diffraction by gratings", Proceedings of the IEE, 73, 894-937, 1985.

8. M. G. Moharam and T. K. Gaylord, "Rigurous coupled-wave analysis of metallic surface-relief gratings ", Journal of the Optical Society of America A, 3, 1780-1796, 1986.

9. M. G. Moharam, E. B. Grann, D. A. Pommet and T. K. Gaylord, "Formulation for stable and efficient implementation of the rigurous coupled-wave analysis of binary gratings" Journal of the Optical Society of America A, 12, 1068-1076, 1995.

10. N. Kamiya, "Rigorous coupled-wave analysis for procatical planar dielectric gratings: 1 . Thickness-changed holograms and some characteristics of diffraction efficiency", Applied Optics, 37, 5843-5853, 1998.

11. P. Dansas and N. Paraire, "Fast modelling of photonic bandgap structures by use of a diffraction-grating approach", Journal of the Optical Society of America A, 15, 1586-1598, 1998.

12. N. Y. Chang and C. J. Juo, "Algorithm based on rigorous coupled-wave analysis for diffractive optical element design", Journal of the Optical Society of America A, 18, 2491-2501, 2001.

13. R. Alferness, "Analysis of propagation at the second-order Bragg angle of a thick holographic grating", Journal of the Optical Society of America, 66, 353-362, 1976.

14. C. Neipp. M. L. Alvarez, S. Gallego, M. Ortuno, J. T. Sheridan, I. Pascual and A. Beléndez, "Angular responses of the first diffracted order in over modulated diffraction gratings", Journal of Modern Optics, accepted.

15. H. I. Bjelkhagen, Silver-Halide Recording Materials, Springer Verlag, Berlin, 1995.

16. G. Zhao, P. Mouroulis, "Diffusion model of hologram formation in dry photopolymer materials", Journal of Modern Optics, 41, 1929-1939, 1994.

17. V. L. Colvin, R. G. Larson, A. L. Harris, M. L. Schilling, "Quantitative model of volume hologram formation in photopolymers", Journal of Applied Physics, 81, 5913-5923, 1997.

18. I. Aubrecht. M. Miler, I. Koudela, "Recording of holographic diffraction gratings in photopolymers: theoretical modelling and real-time monitoring of grating growth", Journal of Modern Optics, 45, 1465-1477, 1998.

19. J. H. Kwon, H. C. Chang and K. C. Woo, "Analysis of temporal behavior of beams diffracted by volume gratings formed in photopolymers", Journal of the Optical Society of America B, 16, 1651-1657, 1999. 
20. G. M. Karpov, V. V. Obukhovsky, T. N. Smirnova, V. V. Lemeshko, "Spatial transfer of matter as a method of holographic recording in photoformers", Optics Communications, 174, 391-404, 2000.

21. J. T. Sheridan, J. R. Lawrence, "Non-local response diffusion model of holographic recording in photopolymer", Journal of the Optical Society of America A, 17, 1108-1114, 2000.

22. J. T. Sheridan, M. Downey, F. T. O'Neill, "Diffusion based model of holographic grating formation in photopolymers: Generalised non-local material responses", Journal of Optics A: Pure and Applied Optics, 3, 477$488,2001$.

23. J. R. Lawrence, F. T. O’Neill, J. T. Sheridan, “Adjusted intensity non-local diffusion model of photopolymer grating formation", Journal of the Optical Society of America B, 19, 621-629, 2002.

24. C. Neipp, A. Beléndez, S. Gallego, M. Ortuño, I. Pascual and J. T. Sheridan, "Angular responses of the first and second diffracted orders in transmission diffraction grating recorded on photopolymer material", Optics Express, 11 (16), 1835-1843, 2003.

25. C. Neipp, M. L. Alvarez, S. Gallego, M. Ortuno, I. Pascual and A. Beléndez, "Comparison between a Thin Matrix Decomposition Method and the Rigorous Coupled Wave Theory applied to volume diffraction gratings", Optik, accepted. 